\title{
A new centrosaurine from the Late Cretaceous of Alberta, Canada, and the evolution of parietal ornamentation in horned dinosaurs
}

Andrew A. Farke, Michael J. Ryan, Paul M. Barrett, Darren H. Tanke, Dennis R. Braman, Mark A. Loewen, and Mark R. Graham

Acta Palaeontologica Polonica 56 (4), 2011: 691-702 doi: http://dx.doi.org/10.4202/app.2010.0121

In 1916, a centrosaurine dinosaur bonebed was excavated within the Campanian-aged deposits of what is now Dinosaur Provincial Park, Alberta, Canada. Specimens from this now-lost quarry, including two parietals, a squamosal, a skull missing the frill, and an incomplete dentary, were purchased by The Natural History Museum, London. The material was recently reprepared and identified herein as a previously unknown taxon, Spinops sternbergorum gen. et sp. nov. Based upon the available locality data and paleopalynology, the quarry lies in either the upper part of the Oldman Formation or the lower part of the Dinosaur Park Formation. The facial region of the partial skull is similar to putative mature specimens of Centrosaurus spp. and Styracosaurus albertensis, with short, rounded postorbital horncores and a large, erect nasal horncore. Parietal ornamentation is consistent on both known parietals and is unique among ceratopsids. Bilateral, procurved parietal hooks occupy the P1 (medial-most) position on the dorsal surface of the parietal and are very similar to those seen in Centrosaurus apertus. Epiparietals in the P2 or possibly P3 position (lateral to P1) manifest as extremely elongate, caudally directed spikes, unlike the condition in $C$. apertus, $S$. albertensis, or any other "derived" centrosaurine. Cladistic analysis suggests that $S$. sternbergorum is closely related to Centrosaurus and Styracosaurus. Historically, based upon the condition in Styracosaurus and related centrosaurines, it was assumed that the medial-most elongated spikes on centrosaurine parietals correspond to the P3 epiparietal position. The exception illustrated in the new taxon suggests that homologies of epiparietals among basal centrosaurines (e.g., Albertaceratops and Diabloceratops ) and derived centrosaurines (e.g., Styracosaurus and "pachyrhinosaurs") should be reconsidered. The medially-placed, caudally-directed "P3" process of basal centrosaurines may, in fact, be homologous with P2.

Key words: Dinosauria, Ornithischia, Centrosaurinae, Ceratopsidae, epiparietal, Oldman Formation, Dinosaur Park Formation, Campanian, Cretaceous, Alberta, Canada.

Andrew A. Farke [afarke@webb.org], Raymond M. Alf Museum of Paleontology, 1175 West Baseline Road, Claremont, CA 91711, USA; Michael J. Ryan [mryan@cmnh.org 
], Department of Vertebrate Paleontology, Cleveland Museum of Natural History, 1 Wade Oval Drive, Cleveland, OH 44106, USA; Paul M. Barrett [p.barrett@nhm.ac.uk

], Department of Palaeontology, Natural History Museum, Cromwell Road, London SW7 5BD, UK; Darren H. Tanke [darren.tanke@gov.ab.ca] and

Dennis R. Braman [dennis.braman@gov.ab.ca], Royal Tyrrell Museum of Palaeontology, P.O. Box 7500, Drumheller, AB T0J 0Y0, Canada; Mark A.

Loewen [mloewen@nhmu.utah.edu], Department of Geology and Geophysics, University of Utah; Natural History Museum of Utah, 301 Wakara Way, Salt

Lake City, UT 84108, USA; Mark R. Graham [m.graham@nhm.ac.uk], Department of Palaeontology, Natural History Museum, Cromwell Road, London SW7 5BD, UK.

This is an open-access article distributed under the terms of the Creative Commons Attribution License (for details please see creativecommons.org), which permits unrestricted use, distribution, and reproduction in any medium, provided the original author and source are credited.

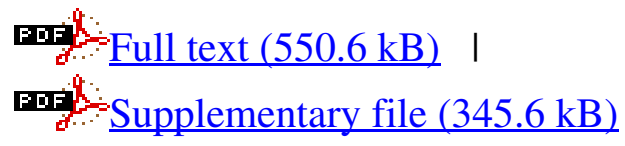

